\title{
Storage Life Prediction for Accelerometer based on Nonlinear Degradation Model
}

\author{
Li Rui, Wang Lixin, Wang Qi, Li Can \\ 304 Teaching and Research Section, The Artillery Engineering University \\ Xi'an 710025, China \\ Lz_zeskr@163.com
}

\begin{abstract}
For the accelerometers which have nonlinear degradation characteristics, the existing linear regression model is difficult to accurately evaluate the storage life. In order to solve this problem, under the condition of the accelerated degradation test, a storage life evaluating method based on nonlinear regression model is advanced. Analyzed the relationship between the characteristic parameters of the model and the stress level, and deduced the life distribution of probability which is restricted by acceleration model, and proposed a statistical analysis method which combines maximum likelihood estimation and parameter identification method. Finally, evaluated a certain type of accelerometer of constant stress accelerated degradation experiment data, and it proved that the validity of the analysis and the method is effective.
\end{abstract}

Keywords-accelerometer; storage life; nonlinear; accelerate model; degradation

\section{INTRODUCTION}

Accelerometers used in some special equipments are usually storage for a long time. Generally, we need to elevate its storage life and reliability so as to supply the basis on device-minding. However, with the continual improvement of method of design and production, its storage life become longer and longer and its reliability become more and more stable. In this conduction, the time and fee spending on collecting historic statistics of its storage life become very great. At the same time, accelerometer as a kind of accurate device, especially some hyper-accurate accelero meter, the cost on acquiring enough statistics is too much. Therefore, using accelerated testing to elevate its storage life and reliability is a better choice.

Accelerated Testing (AT) has two projects, Accelerated Life Testing (ALT) and Accelerated Degradation Testing(ADT). We need to observe the invalid of devices in ALT, so the expense on this method is too high. Meanwhile, ADT can avoid this problem because it only needs the data of degradation. ADT is also divided into two kinds, first is Constant Stress Accelerated Degradation Testing (CSADT), and second is Step Stress Accelerated Degradation Testing (SSADT). Today, CSADT is riper and better accurate in storage life evaluation, while SSADT is still in the stage of development.

At present, researches about ADT main ly point at the degradation whose trace is linear or can be transformed into linear. But the reports about nonlinear degradation process are rarely. And meanwhile, many random process based degradation model presume that diffusion coefficient isn't influenced by temperature stress. However, this supposition conflict with the common sense that equipment becomes more and more unstable with rising temperature. So, this paper proposes a nonlinear degradation model based accelerometer constant stress accelerated degradation testing method in storage condition. At meanwhile, the paper proposed the relationship between temperature stress and parameter such as diffusion coefficient.

\section{ACCELERATION MODEL AND RELIABILITY MODEL}

\section{A. Acceleration Model}

CSADT requires that stress influence the degenerate ratio of device only, but not the degradation mechanis $\mathrm{m}$. Therefore, we should analyze accelero meter's degradation mechanis $m$ in storage condition before evaluating the reliability of accelerometer. Through analysis of FMECA and FTA, literature [1] indicate that accelerometer's degradation trouble mainly focus on the amplifier-circle, oscillator-circle and magnetic Component. Performance degradation of magnetic Component happens in temperature compensation is not enough. In this condition, it has great influence on accelerometer's performance. At the same time, colloid in accelerometer will become ageing because of temperature, and then magnetic assembly will remove. Eventually, accelerometer's performance will degenerate. Consequently, temperature is the main sense stress of accelerometer in storage condition.

When choose temperature stress as main degradation stress of accelerometer in storage condition, we usually apply Arrhenius model to describe relation between function degradation ratio and temperature. Its general expression as:

$$
\theta=A e^{\left(\frac{E}{K S}\right)}
$$

In this equation, $\theta$ is certain life characteristics, $A$ is a constant, $E$ replaces active energy(unit: e V ), $K$ is Boltzmann Constant, $S$ is absolute temperature.

Make logarithm of the equation, we can get another equation below:

$$
\ln \theta=\lambda+\frac{\eta}{s}
$$


Among the equation, $\lambda=\ln A, \eta=E / K$, both of them is undetermined coefficient.

\section{B. Accelerated Degradation Reliable Model Based on Nonlinear Degradation}

At present, most of researches suppose that degradation trace is linear or can be transformed to linear when they build the reliability model in the CSADT. In engineering practice, device's performance degradation trace being nonlinear always happens. In this condition, using linear model will cause evaluation unaccurate. Literature [8] proposed a nonlinear degradation model based on diffusion process. This model can deal with nonlinear degradation process as well as linear degradation process. We can describe this model as below:

$$
X(t)=X(0)+\int_{0}^{t} a(t, \alpha, \beta) d t+\sigma_{b} B(t)
$$

In this equation, $B(t)$ is standard Brownian movement; $X(t)$ replaces degradation process driven by $B(t) ; a(t, \alpha, \beta)$ is drift coefficient of degradation $X(t)$; $\sigma_{b}$ is diffusion coefficient; in $a(t, \alpha, \beta), \alpha$ is random coefficient, reflecting individual difference in the same category and the same batch production. We assume $\alpha \sim N\left(\mu_{\alpha}, \sigma_{\alpha}\right)$ here. $\beta$ is a fixed parameter, reflecting the inherent degradation feature of equipment.

So in the meaning of first-hitting time, and the failure threshold is $w$, The probability density function of the degradation process that described in model (3) is formulated as:

$$
f_{T}(t) \cong E_{\alpha}\left\{\frac{\left(\frac{S_{B}(t)}{t}+\frac{a(t, \alpha, \beta)}{\sigma_{b}}\right)}{\sqrt{2 \pi t}} \exp \left[-\frac{S_{B}{ }^{2}(t)}{2 t}\right]\right\}
$$

In this equation,

$$
S_{B}(t)=\frac{1}{\sigma_{b}}\left(w-\int_{0}^{t} a(\tau, \alpha, \beta) d \tau\right)
$$

Detailed process proving this equation can be found in literature [8]. We extend linear degradation model on the basis of wiener process. Make $a(t, \alpha, \beta)=\alpha \beta t^{\beta-1}$, when $\beta=1$, equation (3) is wiener process; when $\beta \neq 1$, equation (3) is nonlinear degradation process.

Put $a(t, \alpha, \beta)=\alpha \beta t^{\beta-1}$ into equation (4) and equation (5), we can get that:

$$
f_{T}(t) \cong \frac{1}{\sigma_{b} \sqrt{2 \pi t^{3}}} E_{\alpha}\left\{\left(w-\alpha t^{\beta}(1-\beta)\right) \exp \left[-\frac{\left(w-\alpha t^{\beta}\right)^{2}}{2 \sigma_{b}^{2} t}\right]\right\}
$$

Make $X=t^{\beta}(1-\beta), Y=t^{\beta}, Z=\sigma_{b}{ }^{2} t$, we can get that:

$$
\begin{aligned}
& E_{\alpha}\left\{\left(w-\alpha t^{\beta}(1-\beta)\right) \exp \left[-\frac{\left(w-\alpha t^{\beta}\right)^{2}}{2 \sigma_{b}{ }^{2} t}\right]\right\} \\
= & w E_{\alpha}\left\{\exp \left[-\frac{(w-\alpha Y)^{2}}{2 Z}\right]\right\}-X E_{\alpha}\left\{\alpha \exp \left[-\frac{(w-\alpha Y)^{2}}{2 Z}\right]\right\} \\
= & w \sqrt{\frac{Z}{Y^{2} \sigma_{\alpha}{ }^{2}+Z}} \exp \left(-\frac{\left(w-Y \mu_{\alpha}\right)^{2}}{2\left(Y^{2} \sigma_{\alpha}{ }^{2}+Z\right)}\right)- \\
& X \zeta \sqrt{\frac{Z}{Y^{2} \sigma_{\alpha}{ }^{2}+Z}} \exp \left(-\frac{\left(w-Y \mu_{\alpha}\right)^{2}}{2\left(Y^{2} \sigma_{\alpha}{ }^{2}+Z\right)}\right) \\
= & (w-X \zeta) \exp \left(-\frac{\left(w-Y \mu_{\alpha}\right)^{2}}{2\left(Y^{2} \sigma_{\alpha}{ }^{2}+Z\right)}\right)
\end{aligned}
$$

In this equation, $\zeta=\frac{w Y \sigma_{\alpha}{ }^{2}+\mu_{\alpha} Z}{Y^{2} \sigma_{\alpha}{ }^{2}+Z}$.

So equation (6) can be described as:

$$
\begin{aligned}
& f_{T}(t) \cong \frac{1}{\sqrt{2 \pi t^{3}\left(\sigma_{\alpha}{ }^{2} t^{2 \beta-1}+\sigma_{b}^{2}\right)}}\left(w-\left(t^{\beta}-\beta t^{\beta}\right)\right. \\
& \left.\frac{w \sigma_{\alpha}{ }^{2} t^{\beta-1}+\mu_{\alpha} \sigma_{b}^{2}}{\sigma_{\alpha}{ }^{2} t^{2 \beta-1}+\sigma_{b}{ }^{2}}\right) \exp \left[-\frac{\left(w-\mu_{\alpha} t^{\beta}\right)^{2}}{2 t\left(\sigma_{\alpha}{ }^{2} t^{2 \beta-1}+\sigma_{b}{ }^{2}\right)}\right]
\end{aligned}
$$

In equation (8), we careless the effects of temperature stress. When temperature stress is considered, we should analyze the relationship between temperature stress and each parameter. Through analysis, in accelerated degradation testing, temperature stress has an influence on mean of drift coefficient, standard deviation of drift coefficient and diffusion coefficient. But device's feature parameter keep fixed, the reasons are as bellows:

First, the mean of drift coefficient $\mu_{\alpha}$ reflects the speed of performance degenerate. When temperature stress is higher, the degradation will be more aggravated. Therefore, temperature stress $S$ has an influence on the mean of drift coefficient $\mu_{\alpha}$.

Second, Temperature stress $S$ affects drift coefficient's standard deviation $\sigma_{\alpha}$, we can testify it as bellow:

Suppose under the influence of temperature stress, the mean of drift coefficient of $\mathrm{N}$ sets of devices are $\mu_{\alpha}{ }^{\text {si }}$, drift coefficient's standard deviation is $\sigma_{\alpha}{ }^{\text {si }}$. We can get:

$$
\sigma_{\alpha}^{s i}=\sqrt{\frac{\sum_{j=1}^{N}\left(\alpha_{j}^{s i}-\mu_{\alpha}^{s i}\right)^{2}}{N}}
$$

To get a general conclusion, while $i=1,2$, make $\Delta_{j}=a_{j}^{s 2}-a_{j}^{s 1}$, we can get: 


$$
\begin{aligned}
\sigma_{\alpha}^{s 2} & =\sqrt{\frac{1}{N} \sum_{j=1}^{N}\left(\alpha_{j}^{s 2}-\mu_{\alpha}^{s 2}\right)^{2}} \\
& =\sqrt{\frac{1}{N} \sum_{j=1}^{N}\left(\left(\alpha_{j}^{s 1}+\Delta_{j}\right)-\frac{1}{N} \sum_{j=1}^{N}\left(\alpha_{j}^{s 1}+\Delta_{j}\right)\right)^{2}} \\
& =\sqrt{\frac{1}{N} \sum_{j=1}^{N}\left\{\left(\alpha_{j}^{s 1}-\frac{1}{N} \sum_{j=1}^{N} \alpha_{j}^{s 1}\right)+\Psi\right\}^{2}} \\
& =\sqrt{\left(\sigma_{\alpha}^{s 1}\right)^{2}+\frac{2}{N} \sum_{j=1}^{N}\left(\alpha_{j}^{s 1}-\mu_{\alpha}^{s 1}\right) \Psi+\frac{1}{N} \sum_{j=1}^{N} \Psi^{2}}
\end{aligned}
$$

Among this equation, $\Psi=\alpha_{j}^{s 1}+\Delta_{j}$. From the upper equation, we can see that $\alpha_{j}^{s 1}$ isn't equal to $\alpha_{j}^{s 2}$ constantly. It shows temperature stress $S$ affects drift coefficient's standard deviation $\sigma_{\alpha}$.

Third, to calculate briefly, influence of temperature stress on diffusion coefficient $\sigma_{b}$ is neglected in many study. For examp le, literature [6] and literature [7] both suppose that diffusion coefficient is fixed in entire accelerated degradation testing. However, this supposing conflict with the fact. When temperature stress is increasing, device's inner co mponent becoming more unstable, device's output also becoming unstable. So diffusion ratio of function parameter $X(t)$ will become larger.

Forth, equipment characteristic parameter $\beta$ reflects invalid mechanism in degradation process. Depend on research of literature [1] and [8], when equipment's degenerated mechanism isn't change in degradation process, then $\beta$ is fixed.

Through upper analysis, combined with accelerated degradation model, we can get the relationship between drift coefficient's mean $\mu_{\alpha}$, drift coefficient's standard deviation $\sigma_{\alpha}$, diffusion coefficient $\sigma_{b}$ and temperature stress $S$.

$$
\begin{aligned}
& \ln \mu_{\alpha}(s)=\lambda_{\mu \alpha}+\frac{\eta_{\mu \alpha}}{s} \\
& \ln \sigma_{\alpha}(s)=\lambda_{\sigma \alpha}+\frac{\eta_{\sigma \alpha}}{s} \\
& \ln \sigma_{b}(s)=\lambda_{\sigma b}+\frac{\eta_{\sigma b}}{s}
\end{aligned}
$$

Considering life probability distribution function of temperature stress $S$ can be described as follow:

$$
\begin{aligned}
& f_{T}(t, s) \stackrel{\sim}{=} \frac{1}{\sqrt{2 \pi t^{3}\left(\sigma_{\alpha}(s)^{2} t^{2 \beta-1}+\sigma_{b}(s)^{2}\right)}}\left(w-\left(t^{\beta}-\beta t^{\beta}\right)\right. \\
& \left.\frac{w \sigma_{\alpha}(s)^{2} t^{\beta-1}+\mu_{\alpha}(s) \sigma_{b}(s)^{2}}{\sigma_{\alpha}(s)^{2} t^{2 \beta-1}+\sigma_{b}(s)^{2}}\right) \exp \left[-\frac{\left(w-\mu_{\alpha}(s) t^{\beta}\right)^{2}}{2 t\left(\sigma_{\alpha}(s)^{2} t^{2 \beta-1}+\sigma_{b}(s)^{2}\right)}\right]
\end{aligned}
$$

Expectation of life and reliability function $E_{T}(t, s)$ can be calculated as follow:

$$
\begin{aligned}
& E_{T}(s)=\int_{0}^{+\infty} \tau f_{T}(\tau, s) d \tau \\
& R(t, s)=1-\int_{0}^{t} f_{T}(\tau, s) d \tau
\end{aligned}
$$

\section{PARAMETER IDENTIFICATION}

To identify unknown parameter in degradation model, this article combined maximum likelihood estimation and statistical analysis. Supposed N sets of devices are stored in the condition of temperature stress $S$, measure these devices at the same time at the moment of $t_{1}, t_{2} \cdots t_{m}$. The equipment's performance at $t_{j}$ minute can be expressed as:

$$
X_{i}\left(t_{j}, s\right)=\alpha_{i}(s) t_{j}^{\beta}+\sigma_{b}(s) B\left(t_{j}\right)
$$

$\alpha_{i}(s)$ are independent and have the same distribution, accord with $N\left(\mu_{\alpha}(s), \sigma_{\alpha}(s)\right)$, the relationship between each parameter and temperature stress can be found in equation(11).

If directly use maximum likelihood estimation to identify parameter $\Theta=\left(\lambda_{\mu \alpha}, \eta_{\mu \alpha}, \lambda_{\sigma \alpha}, \eta_{\sigma \alpha}, \lambda_{\sigma b}, \eta_{\sigma b}, \beta\right)$, there will be seven unknown parameters. This method cannot be operated actually. So, at first we can suppose temperature stress $S$ is fixed. Using maximum likelihood estimation to evaluate $\Theta(s)=\left(\mu_{\alpha}(s), \sigma_{\alpha}(s), \sigma_{b}(s), \beta \mid s\right)$. Then, as to the maximum likelihood estimated parameter $\hat{\Theta}(s)$ in restriction of different temperature stress, use least square method to identify $\Theta^{\prime}=\left(\lambda_{\mu \alpha}, \eta_{\mu \alpha}, \lambda_{\sigma \alpha}, \eta_{\sigma \alpha}, \lambda_{\sigma b}, \eta_{\sigma b}\right)$. The process is as follows:

The first step:

To certain stress $S$, make $X_{i}=\left(x_{i}\left(t_{1}, s\right), x_{i}\left(t_{2}, s\right), \cdots, x_{i}\left(t_{m}, s\right)\right)^{\prime} \quad, \quad \Gamma=\left(T_{1}, T_{2}, \cdots, T_{N}\right)^{\prime} \quad$, $T_{j}=t_{j}^{\beta}$, so $X_{i}$ follow Gaussian distribution with average being $\mu(s)$ and its variance being $\Sigma(s)$.

$$
\mu(s)=\mu_{\alpha}(s) \Gamma, \quad \sum(s)=\Lambda(s)+\sigma_{\alpha}(s)^{2} \Gamma \Gamma^{\prime}
$$

$$
\Lambda(s)=\sigma_{b}(s)^{2}\left[\begin{array}{cccc}
t_{1} & t_{1} & \cdots & t_{1} \\
t_{1} & t_{2} & \cdots & t_{2} \\
\vdots & \vdots & \ddots & \vdots \\
t_{1} & t_{2} & \cdots & t_{m}
\end{array}\right]
$$

Measured values are independent between different equipment, so unknown parameter $\Theta(s)$ 's logarithm likelihood function can be expressed:

$$
\begin{aligned}
L(\Theta(s) \mid X)= & -\frac{\ln (2 \pi)}{2} N m-\frac{1}{2} N \ln |\Sigma(s)|- \\
& \frac{1}{2} \sum_{i=1}^{N}\left(X_{i}-\mu_{\alpha}(s) \Gamma\right)^{\prime} \Sigma(s)^{-1}\left(X_{i}-\mu_{\alpha}(s) \Gamma\right)
\end{aligned}
$$

Among this equation,

$$
|\Sigma(s)|=|\Lambda(s)|\left(1+\sigma_{\alpha}(s)^{2} \Gamma^{\prime} \Lambda(s)^{-1} \Gamma\right)
$$




$$
\Sigma(s)^{-1}=\Lambda(s)^{-1}-\frac{\sigma_{\alpha}(s)^{2} \Lambda(s)^{-1} \Gamma \Gamma^{\prime} \Lambda(s)^{-1}}{1+\sigma_{\alpha}(s)^{2} \Gamma^{\prime} \Lambda(s)^{-1} \Gamma}
$$

$L(\Theta(s) \mid X)$ Strives for the partial derivative of $\mu_{\alpha}(s)$ and $\sigma_{\alpha}(s)$, we can get that:

$$
\begin{gathered}
\frac{\partial L(\Theta(s) \mid X)}{\partial \mu_{\alpha}(s)}=\frac{\sum_{i=1}^{N} \Gamma^{\prime} \Lambda(s)^{-1} X_{i}-N \mu_{\alpha}(s) \Gamma^{\prime} \Lambda(s)^{-1} \Gamma}{1+\sigma_{\alpha}(s)^{2} \Gamma^{\prime} \Lambda(s)^{-1} \Gamma} \\
\quad \frac{\partial L(\Theta(s) \mid X)}{\partial \sigma_{\alpha}(s)}=\frac{1}{\left(1+\sigma_{\alpha}(s)^{2} \Gamma^{\prime} \Lambda(s)^{-1} \Gamma\right)^{2}} \times \\
\left\{\sum_{i=1}^{N}\left(X_{i}-\mu_{\alpha}(s) \Gamma\right)^{\prime} \Lambda(s)^{-1} \Gamma \Gamma^{\prime} \Lambda(s)^{-1}\left(X_{i}-\mu_{\alpha}(s) \Gamma\right)-\right. \\
\left.N \Gamma^{\prime} \Lambda(s)^{-1} \Gamma\left(\sigma_{\alpha}(s)+\sigma_{\alpha}(s)^{3} \Gamma^{\prime} \Lambda(s)^{-1} \Gamma\right)\right\}
\end{gathered}
$$

Make formula (21) and formula (22) equal to zero, we can get maximum likelihood expression of $\mu_{\alpha}(s)$ and $\sigma_{\alpha}(s)$.

$$
\begin{aligned}
& \hat{\mu}_{\alpha}(s)=\frac{\sum_{i=1}^{N} \Gamma^{\prime} \Lambda(s)^{-1} X_{i}}{N \Gamma^{\prime} \Lambda(s)^{-1} \Gamma} \\
& \hat{\sigma}_{\alpha}(s)= \\
& \left(\frac{\sum_{i=1}^{N}\left(X_{i}-\mu_{\alpha}(s) \Gamma\right){ }^{\prime} \Lambda(s)^{-1} \Gamma^{\prime} \Lambda(s)^{-1}\left(X_{i}-\mu_{\alpha}(s) \Gamma\right)}{N\left(\Gamma^{\prime} \Lambda(s)^{-1} \Gamma\right)^{2}}-\frac{1}{\Gamma^{\prime} \Lambda(s)^{-1} \Gamma}\right)^{\frac{1}{2}}
\end{aligned}
$$

Substitute Eq.23) and Eq.24) into Eq.(18), we can get maximum likelihood function about $\sigma_{b}(s)$ and $\beta$.

$$
\begin{gathered}
L\left(\Theta(s) \mid X, \hat{\mu}_{\alpha}(s), \sigma_{\alpha}(s)\right)=-\frac{N m \ln (2 \pi)}{2}-\frac{N \ln |\Lambda(s)|}{2}- \\
\frac{N}{2}-\frac{1}{2}\left\{\sum_{i=1}^{N} X_{i}^{\prime} \Lambda(s)^{-1} X_{i}-\frac{\sum_{i=1}^{N}\left(\Gamma^{\prime} \Lambda(s)^{-1} X_{i}\right)^{2}}{\Gamma^{\prime} \Lambda(s)^{-1} \Gamma}\right\}- \\
\frac{N}{2} \ln \left\{\frac{N \sum_{i=1}^{N}\left(\Gamma^{\prime} \Lambda(s)^{-1} X_{i}\right)^{2}-\left(\sum_{i=1}^{N} \Gamma^{\prime} \Lambda(s)^{-1} X_{i}\right)^{2}}{N^{2} \Gamma^{\prime} \Lambda(s)^{-1} \Gamma}\right\}
\end{gathered}
$$

At last, using the planar search to Eq.(25), we can get $\sigma_{b}(s)$ and $\beta$ 's maximum likelihood estimated value $\hat{\sigma}_{b}(s)$ and $\hat{\beta}$. Substituting them into Eq.(23) and Eq.(24), we can get $\hat{\mu}_{\alpha}(s)$ and $\hat{\sigma}_{\alpha}(s)$.

The second step:

Corresponding to different temperature stress $S_{l}, l=1 \cdots k$, estimate restricted maximum likihood parameter value $\hat{\Theta}\left(s_{l}\right)$. Substituting $\left(1 / s_{l}, \mu_{\alpha}\left(s_{l}\right)\right)$, $\left(1 / s_{l}, \sigma_{\alpha}\left(s_{l}\right)\right),\left(1 / s_{l}, \sigma_{b}\left(s_{l}\right)\right)$, use least square method to identify $\Theta^{\prime}=\left(\lambda_{\mu \alpha}, \eta_{\mu \alpha}, \lambda_{\sigma \alpha}, \eta_{\sigma \alpha}, \lambda_{\sigma b}, \eta_{\sigma b}\right)$. The shorting of this method is that parameter's identification accuracy may worsen if the quantity of temperature stress is smaller.

\section{ACCELEROMETER STORAGE LIFETIME AND RELIABILITY EVALUATION}

This section will evaluate storage lifetime and reliability of pendulous accelerometer beamed by jewel bearing. To this accelerometer, on the purpose of estimating its function performance, we usually evaluate the first degree's scale factor $K_{1}$ which influence output more.

At the temperature of $65^{\circ} \mathrm{C} 、 75^{\circ} \mathrm{C}$ and $85^{\circ} \mathrm{C}$,

\begin{tabular}{|c|c|c|c|c|c|c|}
\hline $\begin{array}{c}\text { Time } \\
\text { (hours) }\end{array}$ & $D_{k 1} 2$ & $D_{k 1} 2$ & $D_{k 1} 3$ & $D_{k 1} 4$ & $D_{k 1} 5$ & $D_{k 1} 6$ \\
\hline 1227.8 & $\begin{array}{c}3.112 \\
\text { E-04 }\end{array}$ & $\begin{array}{c}1.071 \\
\text { E-04 }\end{array}$ & $\begin{array}{c}1.523 \\
\text { E-04 }\end{array}$ & $\begin{array}{c}1.505 \\
\text { E-04 }\end{array}$ & $\begin{array}{c}1.221 \\
\text { E-04 }\end{array}$ & $\begin{array}{c}7.269 \\
\text { E-05 }\end{array}$ \\
\hline 1539.8 & $\begin{array}{c}9.693 \\
\text { E-05 }\end{array}$ & $\begin{array}{c}4.591 \\
\text { E-05 }\end{array}$ & $\begin{array}{c}8.677 \\
\text { E-05 }\end{array}$ & $\begin{array}{c}5.990 \\
\text { E-05 }\end{array}$ & $\begin{array}{c}4.226 \\
\text { E-05 }\end{array}$ & $\begin{array}{c}3.507 \\
\text { E-05 }\end{array}$ \\
\hline 1851.8 & $\begin{array}{c}2.755 \\
\text { E-04 }\end{array}$ & $\begin{array}{c}8.162 \\
\text { E-05 }\end{array}$ & $\begin{array}{c}9.208 \\
\text { E-05 }\end{array}$ & $\begin{array}{c}1.191 \\
\text { E-04 }\end{array}$ & $\begin{array}{c}1.035 \\
\text { E-04 }\end{array}$ & $\begin{array}{c}5.088 \\
\text { E-05 }\end{array}$ \\
\hline 2475.8 & $\begin{array}{c}7.142 \\
\text { E-05 }\end{array}$ & $\begin{array}{c}7.142 \\
\text { E-05 }\end{array}$ & $\begin{array}{c}2.089 \\
\text { E-04 }\end{array}$ & $\begin{array}{c}9.091 \\
\text { E-05 }\end{array}$ & $\begin{array}{c}4.568 \\
\text { E-05 }\end{array}$ & $\begin{array}{c}7.078 \\
\text { E-05 }\end{array}$ \\
\hline 2787.8 & $\begin{array}{c}4.489 \\
\text { E-04 }\end{array}$ & $\begin{array}{c}1.275 \\
\text { E-04 }\end{array}$ & $\begin{array}{c}1.912 \\
\text { E-04 }\end{array}$ & $\begin{array}{c}2.052 \\
\text { E-04 }\end{array}$ & $\begin{array}{c}1.708 \\
\text { E-04 }\end{array}$ & $\begin{array}{c}9.404 \\
\text { E-05 }\end{array}$ \\
\hline 3099.8 & $\begin{array}{c}2.296 \\
\text { E-04 }\end{array}$ & $\begin{array}{c}3.265 \\
\text { E-04 }\end{array}$ & $\begin{array}{c}8.322 \\
\text { E-05 }\end{array}$ & $\begin{array}{c}1.374 \\
\text { E-04 }\end{array}$ & $\begin{array}{c}1.191 \\
\text { E-04 }\end{array}$ & $\begin{array}{c}7.063 \\
\text { E-05 }\end{array}$ \\
\hline 3411.8 & $\begin{array}{c}2.653 \\
\text { E-04 }\end{array}$ & $\begin{array}{c}2.194 \\
\text { E-04 }\end{array}$ & $\begin{array}{c}3.541 \\
\text { E-06 }\end{array}$ & $\begin{array}{c}1.101 \\
\text { E-04 }\end{array}$ & $\begin{array}{c}1.116 \\
\text { E-04 }\end{array}$ & $\begin{array}{c}3.924 \\
\text { E-05 }\end{array}$ \\
\hline 3716.5 & $\begin{array}{c}3.724 \\
\text { E-04 }\end{array}$ & $\begin{array}{c}1.326 \\
\text { E-04 }\end{array}$ & $\begin{array}{c}2.833 \\
\text { E-04 }\end{array}$ & $\begin{array}{c}2.097 \\
\text { E-04 }\end{array}$ & $\begin{array}{c}1.535 \\
\text { E-04 }\end{array}$ & $\begin{array}{c}1.161 \\
\text { E-04 }\end{array}$ \\
\hline 4020.5 & $\begin{array}{c}5.050 \\
\text { E-04 }\end{array}$ & $\begin{array}{c}2.959 \\
\text { E-04 }\end{array}$ & $\begin{array}{c}1.948 \\
\text { E-04 }\end{array}$ & $\begin{array}{c}2.464 \\
\text { E-04 }\end{array}$ & $\begin{array}{c}2.099 \\
\text { E-04 }\end{array}$ & $\begin{array}{c}1.156 \\
\text { E-04 }\end{array}$ \\
\hline 4332.5 & $\begin{array}{c}5.101 \\
\text { E-04 }\end{array}$ & $\begin{array}{c}3.801 \\
\text { E-04 }\end{array}$ & $\begin{array}{c}5.153 \\
\text { E-04 }\end{array}$ & $\begin{array}{c}3.515 \\
\text { E-04 }\end{array}$ & $\begin{array}{c}2.438 \\
\text { E-04 }\end{array}$ & $\begin{array}{c}2.153 \\
\text { E-04 }\end{array}$ \\
\hline 4644.5 & $\begin{array}{c}8.264 \\
\text { E-04 }\end{array}$ & $\begin{array}{c}1.056 \\
\text { E-03 }\end{array}$ & $\begin{array}{c}1.197 \\
\text { E-03 }\end{array}$ & $\begin{array}{c}7.349 \\
\text { E-04 }\end{array}$ & $\begin{array}{r}4.747 \\
\text { E-04 }\end{array}$ & $\begin{array}{c}4.965 \\
\text { E-04 }\end{array}$ \\
\hline
\end{tabular}
choose six accelerometers to carry out accelerated degradation testing, cooling accelerometer to normal state $\left(25^{\circ} \mathrm{C}\right.$ ) before testing. Choosing the first testing temperature of each accelerometer as datum mark, calculating relative drift value $D_{k 1}$ of other testing times, and invalid threshold is 0.006 . To brief this question, testing process' influence on accelerometer function degradation is neglected. Detailed testing parameter can be seen in table 1 3. (The early part of the data has been given up because of device's fault)

Table 2 Drift of scale factor $D_{k 1}$ with $s=75^{\circ} \mathrm{C}$

\begin{tabular}{ccccccc}
\hline $\begin{array}{c}\text { Time } \\
\text { (hours) }\end{array}$ & $D_{k 1}{ }^{2}$ & $D_{k 1} 2$ & $D_{k 1} 3$ & $D_{k 1} 4$ & $D_{k 1} 5$ & $D_{k 1} 6$ \\
\hline \multirow{2}{*}{500.0} & 1.683 & 2.092 & 7.505 & 8.752 & 7.480 & 2.221 \\
& E-04 & E-04 & E-06 & E-05 & E-05 & E-05 \\
666.7 & 1.428 & 4.030 & 1.088 & 1.402 & 1.150 & 6.849 \\
& E-04 & E-04 & E-04 & E-04 & E-04 & E-05 \\
& 1.939 & 1.888 & 1.651 & 9.197 & 8.990 & 6.342 \\
833.3 & E-04 & E-04 & E-04 & E-05 & E-05 & E-05
\end{tabular}




\begin{tabular}{|c|c|c|c|c|c|c|}
\hline 1083.3 & $\begin{array}{l}1.530 \\
\text { E-05 }\end{array}$ & $\begin{array}{l}5.050 \\
\text { E-04 }\end{array}$ & $\begin{array}{c}7.505 \\
\text { E-05 }\end{array}$ & $\begin{array}{c}1.448 \\
\text { E-04 }\end{array}$ & $\begin{array}{c}1.051 \\
\text { E-04 }\end{array}$ & $\begin{array}{c}6.891 \\
\text { E-05 }\end{array}$ \\
\hline 1333.3 & $\begin{array}{c}2.041 \\
\text { E-05 }\end{array}$ & $\begin{array}{c}3.316 \\
\text { E-04 }\end{array}$ & $\begin{array}{c}1.051 \\
\text { E-04 }\end{array}$ & $\begin{array}{c}9.889 \\
\text { E-05 }\end{array}$ & $\begin{array}{c}7.599 \\
\text { E-05 }\end{array}$ & $\begin{array}{c}6.053 \\
\text { E-05 }\end{array}$ \\
\hline 2083.3 & $\begin{array}{c}5.510 \\
\text { E-04 }\end{array}$ & $\begin{array}{l}1.530 \\
\text { E-04 }\end{array}$ & $\begin{array}{c}1.219 \\
\text { E-04 }\end{array}$ & $\begin{array}{c}1.431 \\
\text { E-04 }\end{array}$ & $\begin{array}{c}1.529 \\
\text { E-04 }\end{array}$ & $\begin{array}{c}4.846 \\
\text { E-05 }\end{array}$ \\
\hline 2333.3 & $\begin{array}{c}2.908 \\
\text { E-04 }\end{array}$ & $\begin{array}{c}4.030 \\
\text { E-04 }\end{array}$ & $\begin{array}{c}6.191 \\
\text { E-05 }\end{array}$ & $\begin{array}{c}1.644 \\
\text { E-04 }\end{array}$ & $\begin{array}{c}1.413 \\
\text { E-04 }\end{array}$ & $\begin{array}{c}5.576 \\
\text { E-05 }\end{array}$ \\
\hline 2583.3 & $\begin{array}{c}1.097 \\
\text { E-03 }\end{array}$ & $\begin{array}{c}5.510 \\
\text { E-04 }\end{array}$ & $\begin{array}{c}2.251 \\
\text { E-04 }\end{array}$ & $\begin{array}{c}3.523 \\
\text { E-04 }\end{array}$ & $\begin{array}{c}3.493 \\
\text { E-04 }\end{array}$ & $\begin{array}{c}1.152 \\
\text { E-04 }\end{array}$ \\
\hline 2833.3 & $\begin{array}{c}9.489 \\
\text { E-04 }\end{array}$ & $\begin{array}{c}1.020 \\
\text { E-05 }\end{array}$ & $\begin{array}{c}5.084 \\
\text { E-04 }\end{array}$ & $\begin{array}{c}1.872 \\
\text { E-04 }\end{array}$ & $\begin{array}{c}2.413 \\
\text { E-04 }\end{array}$ & $\begin{array}{c}1.409 \\
\text { E-04 }\end{array}$ \\
\hline 3083.3 & $\begin{array}{c}7.346 \\
\text { E-04 }\end{array}$ & $\begin{array}{c}9.642 \\
\text { E-04 }\end{array}$ & $\begin{array}{c}6.548 \\
\text { E-04 }\end{array}$ & $\begin{array}{c}4.183 \\
\text { E-04 }\end{array}$ & $\begin{array}{c}3.902 \\
\text { E-04 }\end{array}$ & $\begin{array}{c}2.721 \\
\text { E-04 }\end{array}$ \\
\hline 3333.3 & $\begin{array}{c}1.750 \\
\text { E-03 }\end{array}$ & $\begin{array}{c}2.576 \\
\text { E-03 }\end{array}$ & $\begin{array}{c}9.662 \\
\text { E-04 }\end{array}$ & $\begin{array}{c}1.052 \\
\text { E-03 }\end{array}$ & $\begin{array}{c}9.308 \\
\text { E-04 }\end{array}$ & $\begin{array}{c}5.125 \\
\text { E-04 }\end{array}$ \\
\hline
\end{tabular}

Table 3 Drift of scale factor $D_{k 1}$ with $s=85^{\circ} \mathrm{C}$

\begin{tabular}{ccccccc}
\hline $\begin{array}{c}\text { Time } \\
\text { (hours) }\end{array}$ & $D_{k 1} 2$ & $D_{k 1} 2$ & $D_{k 1} 3$ & $D_{k 1} 4$ & $D_{k 1} 5$ & $D_{k 1} 6$ \\
\hline \multirow{2}{*}{400.0} & 9.183 & 1.275 & 3.921 & 6.984 & 3.431 & 6.734 \\
& E-05 & E-04 & E-05 & E-05 & E-05 & E-05 \\
\multirow{2}{*}{500.0} & 3.316 & 3.469 & 8.256 & 9.896 & 8.274 & 9.055 \\
& E-04 & E-18 & E-06 & E-05 & E-05 & E-05 \\
600.0 & 5.101 & 2.347 & 3.653 & 9.414 & 9.620 & 1.477 \\
& E-05 & E-04 & E-04 & E-05 & E-05 & E-04 \\
700.0 & 2.551 & 8.162 & 1.445 & 1.036 & 9.511 & 1.183 \\
& E-04 & E-05 & E-04 & E-04 & E-05 & E-04 \\
1100.0 & 4.030 & 4.795 & 1.527 & 2.799 & 1.442 & 2.697 \\
& E-04 & E-04 & E-04 & E-04 & E-04 & E-04 \\
1300.0 & 4.285 & 1.071 & 6.811 & 1.636 & 1.221 & 1.575 \\
& E-04 & E-04 & E-05 & E-04 & E-04 & E-04 \\
1900.0 & 1.173 & 4.387 & 3.529 & 1.816 & 1.155 & 2.196 \\
& E-04 & E-04 & E-04 & E-04 & E-04 & E-04 \\
2100.0 & 4.183 & 5.612 & 1.125 & 3.142 & 3.555 & 4.802 \\
& E-04 & E-04 & E-03 & E-04 & E-04 & E-04 \\
2300.0 & 4.234 & 9.438 & 1.523 & 4.439 & 4.516 & 6.627 \\
& E-04 & E-04 & E-03 & E-04 & E-04 & E-04 \\
2500.0 & 1.959 & 2.438 & 1.676 & 1.399 & 9.011 & 1.517 \\
& E-03 & E-03 & E-03 & E-03 & E-04 & E-03 \\
\hline
\end{tabular}

Accelerometer's degradation trace as follows:

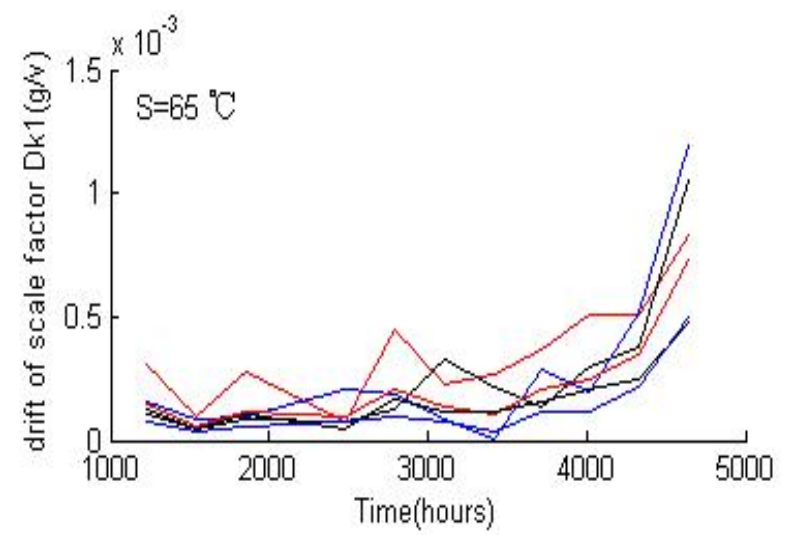

Figure 1. Drift of scale factor $D_{k 1}$ with $s=65^{\circ} \mathrm{C}$

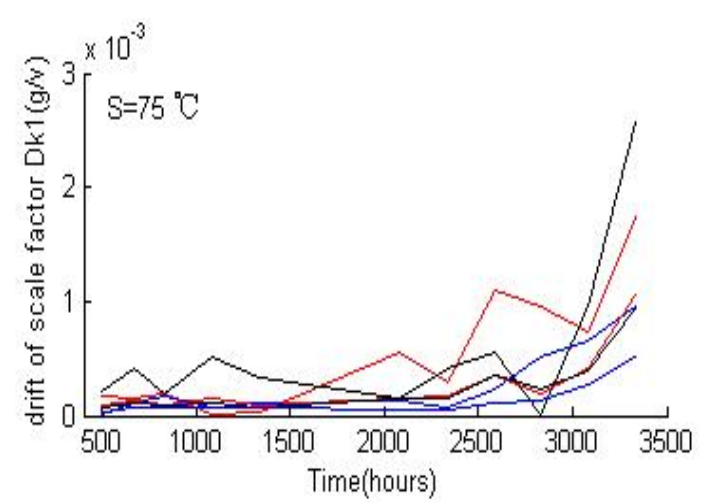

Figure 2. Drift of scale factor $D_{k 1}$ with $s=75^{\circ} \mathrm{C}$

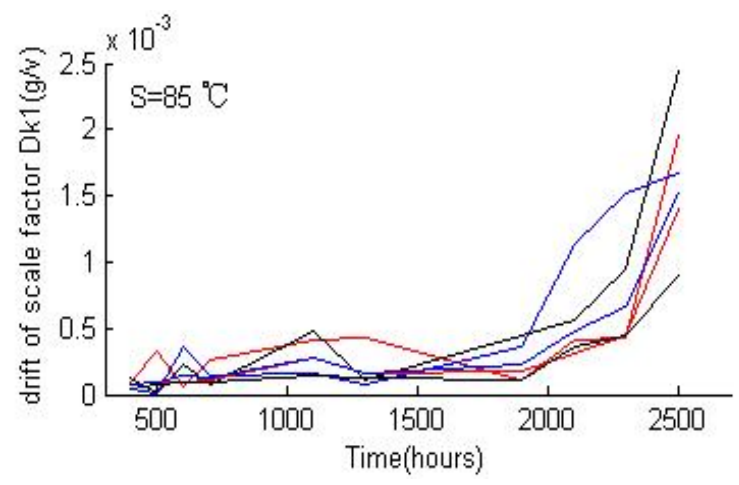

Figure 3. Drift of scale factor $D_{k 1}$ with $s=85^{\circ} \mathrm{C}$

From Fig.1 3, we can see that function degradation trace of this version accelerometer is nonlinear, and its diffusion ratio increases with time. So Eq. (3) are more fit table to describe it. Use the method in section 3 to evaluate parameter in table 1 3. The results list in table 4.

Table 4 Distribute parameter of storage life under stress

\begin{tabular}{ccccc}
\hline \multirow{5}{*}{$S$} & \multicolumn{4}{c}{$\Theta(s)$} \\
\cline { 2 - 5 } & $\hat{\mu}_{\alpha}(s)$ & $\hat{\sigma}_{\alpha}(s)$ & $\hat{\sigma}_{b}(s)$ & $\hat{\beta}$ \\
\hline $65^{\circ} \mathrm{C}$ & $1.167 \mathrm{E}-67$ & $4.263 \mathrm{E}-68$ & $6.027 \mathrm{E}-06$ & 17.375 \\
$75^{\circ} \mathrm{C}$ & $2.502 \mathrm{E}-64$ & $1.513 \mathrm{E}-64$ & $1.280 \mathrm{E}-05$ & 17.201 \\
$85^{\circ} \mathrm{C}$ & $1.881 \mathrm{E}-61$ & $8.452 \mathrm{E}-62$ & $1.464 \mathrm{E}-05$ & 17.012 \\
\hline
\end{tabular}

We can draw a conclusion from table 4 that equipment's inherent feature parameter $\beta$ doesn't change much with stress level. This has proved analys is in section 2.2, and also testified that degradation mechanism of accelerometer doesn't change.

On the basis of $\Theta(s)$, combined with Eq.(11), use least square method to fit $\Theta^{\prime}$, the result list in table 5 .

Table 5 Parameter of accelerate model

\begin{tabular}{cccccc}
\hline \multicolumn{6}{c}{$\Theta(s)$} \\
\hline$\lambda_{\mu \alpha}$ & $\eta_{\mu \alpha}$ & $\lambda_{\sigma \alpha}$ & $\eta_{\sigma \alpha}$ & $\lambda_{\sigma b}$ & $\eta_{\sigma b}$ \\
\hline 3.303 & -1.128 & 1.485 & -5.068 & 1.618 & -5.243 \\
E-60 & E-57 & E-60 & E-58 & E-04 & E-02 \\
\hline
\end{tabular}




\section{Table 6 Distribute parameter of storage life}

\begin{tabular}{ccc}
\multicolumn{3}{c}{$S_{0}=20^{\circ} \mathrm{C}$} \\
\hline$\hat{\mu}_{\alpha}\left(S_{0}\right)$ & $\hat{\sigma}_{\alpha}\left(S_{0}\right)$ & $\hat{\sigma}_{b}\left(S_{0}\right)$ \\
\hline $1.099 \mathrm{E}-84$ & $2.533 \mathrm{E}-85$ & $5.694 \mathrm{E}-07$ \\
\hline
\end{tabular}

So, when accelerometer is stored in normal state ( $s=20^{\circ} \mathrm{C}$ ), we can get accelerometer's storage lifetime distribution parameter (in table 6).

$$
\hat{\beta}=\frac{\hat{\beta}_{1}+\beta_{2}+\beta_{3}}{3}=17.196
$$

Rely on upper parameters, we can calculate lifetime feature of this version accelerometer in storage.

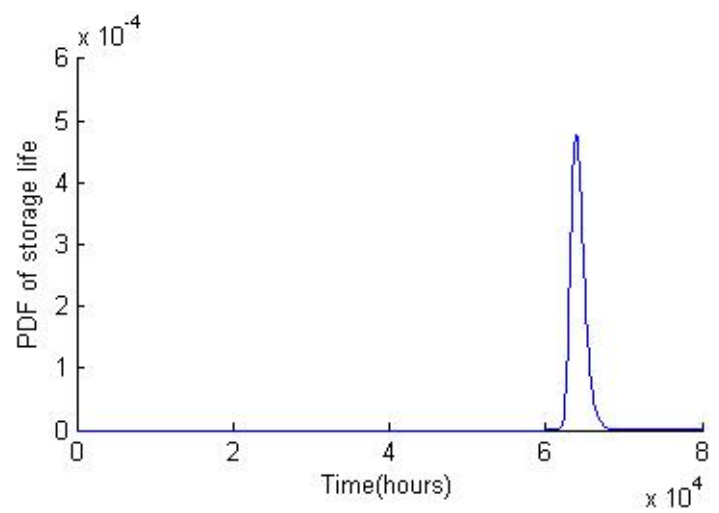

Figure 4. PDF of accelerometer's storage life

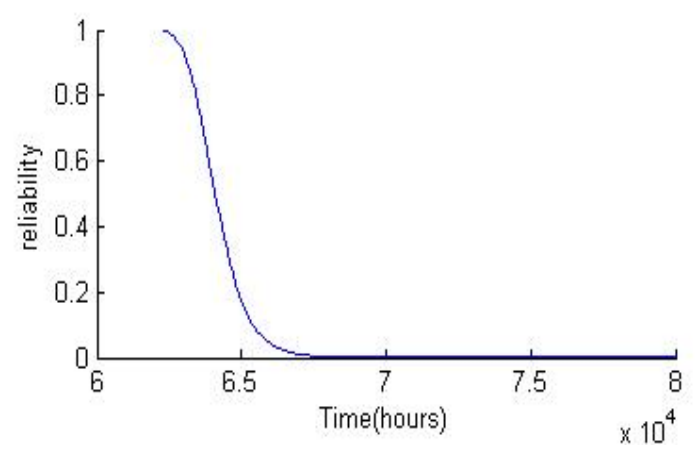

Figure 5. The storage reliability of accelerometer

Finally, through Eq.(13), accelerometer's storage lifetime average is $E_{T}(s)=6.420 \mathrm{E}+04(h)$ 。

\section{SUMMARY}

Based on nonlinear function degradation model, the article has supplied an evaluation method of storage lifetime in accelerated degradation testing condition. Also the composition has discussed stress factor's influence on accelerometer's performance degradation in storage condition, and analyzed parameter influenced by stress in nonlinear degradation model. Through analysis and experiment, in accelerated degradation testing, drift coefficient and diffusion ratio are both influenced by stress, with feature parameter reflecting equipment inherent degradation function being fixed. Through storage lifetime evaluation of accelerometer with nonlinear degradation feature, parameters correspond to analysis value, proving this method being valid. Statistical analysis method is used to identify parameter, so lesser statistics and stress level may lower evaluation accuracy.

\section{REFERENCES}

[1] YUAN Hong-jie, LI Lou-de, DUAN Gang, WU Hao. Storage life and reliability evaluation of accelerometer by step stress accelerated degradation testing [J]. Journal of Chinese Inertial Technology, 2012, 20(1): 113-116.

[2] Si X S, Wang W, Hu C H, Zhou D H. Remaining useful life estimation-A review on the statistical data driven approaches [J]. 2010, European Journal of Operational Research, 2011, 213(1): 114.

[3] Zhao Wenbiao, Elsayed E A. An accelerated life testing model involving performance degradation [C]// 2004 Annual Symposium on Reliability and Maintainability: 324-329.

[4] Jinsuk Lee, Rong Pan. Analyzing step-stress accelerated life testing data using generalized linear models [J]. IIE Transactions, 2010, 42(8): 589-598.

[5] Xu Zhenhong. Storage life prediction methods research of weapons system [D].Harbin, China: Harbin Institute of Technology, 2010.

[6] LI Xiao-yang, JIANG Tong-min. Constant-stress accelerated degradation testing of satellite assemblies [J]. Acta Aeronautic et Astronautic Sinic, 2007,28(Sup.):S100-103.

[7] Meeker W G, Luls A E. Accelerated degradation tests: modeling and analysis [J]. Technometrics,1998,40(2):89-99.

[8] Si X S, Wang W, Hu C H, Zhou D H, Pecht M. Remaining useful life estimation based on a nonlinear diffusion degradation

[9] Process [J]. IEEE Transactions on Reliability, 2012, 61(1): 50-67.

[10] Peng C Y, Tseng S T. Mis-specification analysis of linear degradation models. IEEE Transactions on Reliability, 2009, 58(3): 444-455. 